\title{
Gastrointestinal Bleeding associated with Bowel Perforation by Foreign Body in the Context of a SARS-CoV-2 Infection during Pregnancy
}

\author{
Salvador Jiménez-Chaidez ${ }^{1}$, María Lourdes Gómez-Sousa ${ }^{1}$, Arturo Maximiliano Ruiz-Beltrán², Francisco \\ Javier Ruiloba-Portilla ${ }^{1}$, Fabiola Gallardo-Gómez ${ }^{1}$, Cintia María Sepúlveda-Rivera ${ }^{1}$ \\ ${ }^{1}$ Department of Obstetrics and Gynecology, Instituto Nacional de Perinatología Isidro Espinosa de los Reyes, Mexico City, Mexico; \\ ${ }^{2}$ Department of Internal Medicine, Instituto Nacional de Ciencias Médicas y Nutrición Salvador Zubirán.
}

\section{Corresponding Author:}

Dr Cintia María Sepúlveda-Rivera

Email: cintiia_sepulveda@hotmail.com

This is an Open Access article distributed under the terms of the Creative Commons Attribution License (creativecommons.org/ licenses/by/3.0).

\begin{abstract}
Background: The impact of SARS-CoV-2 infection during pregnancy is currently uncertain, however, maternal respiratory and intestinal symptoms, as well as fetal complications such as abortion, intrauterine growth restriction and premature delivery have been reported. Case Report: A 27-week pregnant lady with a history of Bochdalek hernia corrected at birth presented with hematemesis, hematochezia, and second stage labor. A live newborn was obtained and died 20 minutes after neonatal resuscitation maneuvers. The pan-endoscopy of mother revealed a foreign body in the jejunum. Laparotomy was performed with repair of the diaphragmatic defect and $30 \mathrm{~cm}$ jejunum resection with lateral-lateral anastomosis. Furthermore, a thoracoabdominal tomography revealed the presence of consolidations in the pulmonary lobes, and a PCR for SARSCoV-2 was reported positive. Conclusion: It is not usual to find an intestinal perforation due to migration of the repair mesh of a diaphragmatic hernia, and the pathophysiological mechanisms of SARS-CoV-2 infection that may have influenced the perinatal outcome discussed above are currently unknown.
\end{abstract}

Keywords: COVID-19, Gastrointestinal Hemorrhage, Intestinal Perforation, Pregnancy, SARS-COV-2, Surgical Mesh.

\section{Introduction}

Coronavirus disease 2019 (COVID-19) is considered the biggest pandemic of the century [1]. The main gastrointestinal manifestations include digestive tract bleeding [2]. Fetal complications include preterm delivery. A congenital diaphragmatic hernia is a developmental defect of the posterolateral diaphragm [3]. We hereby report the case of a pregnant patient, with a history of a Bochdalek hernia repaired at birth, SARS-CoV-2 (severe acute respiratory syndrome coronavirus 2 ) infection, and gastrointestinal bleeding.

\section{Case Report}

In July 2020, a 21-year-old primigravida with a history of incomplete situs inversus and a
Bochdalek hernia repaired at birth with a mesh and 27 week pregnancy, attended the emergency department of a third-level care center. She reported post-prandial fullness, precocious satiety, and presented with hematemesis, hematochezia, and uterine contractions. On physical examination, she had paleness, tachycardia, and second stage labor. A non-reactive female product was delivered vaginally, that died after 20 minutes of neonatal resuscitation maneuvers. Additionally, the patient had a hemoglobin of $4.8 \mathrm{~g} / \mathrm{dL}$ upon admission, which was attributed to gastrointestinal bleeding, since transvaginal bleeding during labor was scarce. During her stay in the recovery room, the patient developed hypotension and an altered level of consciousness with a tendency to somnolence, for which she was transferred to the intensive 
care unit. A central venous catheter was placed and fluid resuscitation treatment, blood products, and amines were administered. As an extension study, the thoracoabdominal tomography reported alveolar occupation at the expense of ground glass opacification with a tendency to consolidate with a central and peripheral distribution, irregular morphology, and bilateral involvement of the lower pulmonary lobes, as well as a solution of continuity of the right hemidiaphragm with protrusion of intestinal loops (terminal ileum and ascending colon) in the right lung base and findings suggestive of an intraluminal foreign body in the proximal jejunum segment [Fig.1]. A pan-endoscopy reported an absence of active bleeding. During colonoscopy, hematic remains were visualized in the colonic mucosa, without active bleeding. After the colonoscopy failed to identify the bleeding site, a video capsule endoscopy was performed, through which an image suggestive of a foreign body was observed in the fourth portion of the duodenum and proximal jejunum with mucosal erosion and stigmata of recent hemorrhage [Fig.2].

On the first day of hospital admission, the patient developed fever. With the aforementioned, and the pulmonary findings in the imaging studies, the diagnosis of pneumonia was integrated. Empirical antibiotic treatment based on macrolide and a third-generation cephalosporin was initiated. Subsequently, a polymerase chain reaction for SARS-CoV-2 was carried out, which yielded a positive result. Due to the presence of the foreign body reported in the imaging studies, we decided to surgically intervene the patient. Laparoscopic adhesiolysis was performed and the diaphragmatic defect was repaired with an open approach. The enterotomy site and the foreign body were identified in the fourth portion of the duodenum and the proximal jejunum. A $30 \mathrm{~cm}$ jejunal resection was performed with a lateral-lateral anastomosis with a stapler. She had an adequate post-surgical evolution.

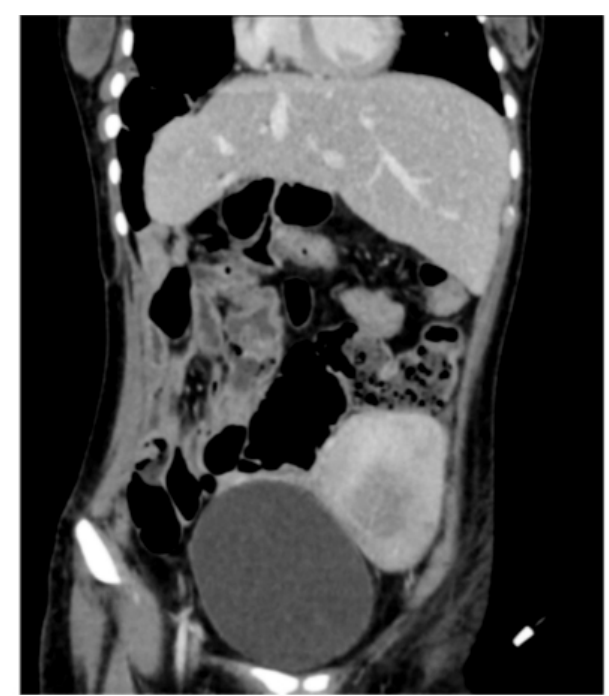

Fig.1: Thoracoabdominal tomography with solution of continuity of the right hemidiaphragm.

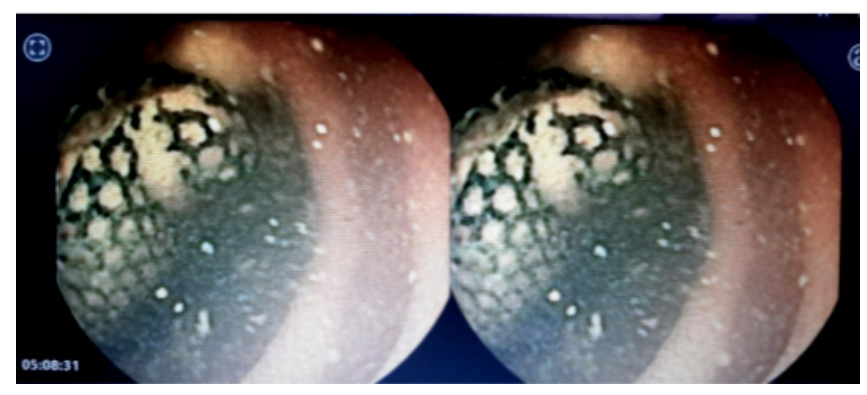

Fig.2: Video capsule endoscopy image suggestive of a foreign body in the fourth portion of the duodenum and proximal jejunum.

\section{Discussion}

We report the case of a pregnant patient with COVID-19 infection, which presented with preterm delivery, pneumonia and gastrointestinal bleeding. Her relevant history included the placement of a mesh for the repair of a Bochdalek hernia at birth. The mesh probably perforated the small intestine at some point in her life without previously generating gastrointestinal bleeding. Her pregnancy and positive COVID-19 status were considered a significant trigger for the lower digestive tract bleeding.

In a study carried out by Takayasu et al. in 2017, the 20-year outcomes of 49 patients 
diagnosed with congenital diaphragmatic hernia and mesh repair at birth were analyzed, reporting the following complications in order of frequency: intestinal obstruction (23\%), gastroesophageal reflux $(9.5 \%)$, and thoracic wall deformities $(2 \%)$; no cases of intestinal perforation were reported due to mesh migration, as in the case of our patient [3]. Li et al. conducted a systematic review in 2019 that analyzed mesh migration in patients with hiatal hernia and subsequent intestinal erosion. A total of 50 cases were studied, the average period between repair and intestinal erosion was 2 years and the material most commonly identified was polypropylene. Furthermore, authors mentioned that intestinal erosion by mesh used during the repair of hernial defects is actually underdiagnosed and scarcely reported in the literature [4].

Speaking about the effect of COVID-19 in the digestive tract, the presence of a lymphocytic inflammatory infiltrate in the esophagus, stomach, colon, and liver has been demonstrated, without apparent damage to the intestinal epithelium. This suggests the reflection of an immune response rather than organic damage [2]. Despite the anticipated increased risk of infection by COVID-19 during pregnancy, the relatively low mortality of this population group has been an area of wonder [5]. Gastrointestinal bleeding during pregnancy is a rare condition and represents a diagnostic challenge for the obstetrician. Endoscopy is the most useful tool for identification, treatment, and prognosis, during or after pregnancy [6].

We developed two possible theories for the explanation of the mechanism of trauma in our patient. The first is that physiological changes during pregnancy, such as the increase in the transverse diameter of the rib cage and the elevation of the diaphragm, produced a sudden tension in the mesh, which could have caused its migration with subsequent intestinal perforation. The other theory is that the mesh may have migrated asymptomatically during childhood, with SARS-CoV-2 infection being the triggering factor for bleeding due to the previously detailed immune reaction.

\section{Conclusion}

Gastrointestinal bleeding during pregnancy is rare. There is no standardized management guide, so the diagnosis and treatment should be applied equally as in a non-pregnant patient. It is not usual to find an intestinal perforation due to migration of the repair mesh of a diaphragmatic hernia, and the pathophysiological mechanisms of SARS-CoV-2 infection that may have influenced the perinatal outcome discussed above are currently unknown.

Contributors: SJ-C: conceptualization, data curation, investigation and writing; MLG-S: supervision and validation; AMR-B: investigation and validation; FJR-P: investigation and writing; FG-G: investigation and writing; CMS-R: conceptualization, data curation, investigation, writing, supervision and validation. CMS-R will act as a study guarantor. All authors approved the final version of this manuscript and are responsible for all aspects of this study.

Funding: Supported by Instituto Nacional de Perinatología Isidro Espinosa de los Reyes; Competing interests: MLG-S is speaker/consultant for Bayer-Mexico. The rest of the authors declare no conflict of interest.

\section{References}

1. Ahmed S, Zimba O, Gasparyan AY. Thrombosis in coronavirus disease 2019 (COVID-19) through the prism of Virchow's triad. Clin Rheumatol. 2020;25:239242.

2. Oba J, Brunow W, Silva C, Delgado A. Gastrointestinal manifestations and nutritional therapy during COVID-19 pandemic: a practical guide for pediatricians. Einstein (São Paulo). 2020;18:eRW5774.

3. Takayasu H, Masumoto K, Jimbo T, Sakamoto N, Sasaki $\mathrm{T}$, Uesugi $\mathrm{T}$, et al. Analysis of risk factors of long-term complications in congenital diaphragmatic hernia: A single institution's experience. Asian J Surg. 2017;40:1-5.

4. Li J, Cheng T. Mesh erosion after hiatal hernia repair: the tip of the iceberg? Hernia. 2019; 23:1243-1252.

5. Vivant A, Vauloup-Fellous C, Prevot S, Zupan V, Suffee $\mathrm{C}$, Do Cao J, et al. Transplacental transmission of SARSCoV-2 infection. Nat Commun. 2020;11:3572.

6. De Lima A, Galjart B, Wisse PH, Bramer WM, van der Woude CJ. Does lower gastrointestinal endoscopy during pregnancy pose a risk for mother and child? A systematic review. BMC Gastroenterol. 2015;15:15. 